\title{
High-z massive galaxies in the Hubble Deep Field South
}

\author{
P. Saracco ${ }^{1}$, M. Longhetti ${ }^{1}$, E. Giallongo ${ }^{2}$, S. Arnouts ${ }^{3}$, S. Cristiani ${ }^{4}$, S. D’Odorico ${ }^{5}$, \\ A. Fontana ${ }^{2}$, M. Nonino ${ }^{4}$, and E. Vanzella ${ }^{5,6}$ \\ 1 INAF - Osservatorio Astronomico di Brera, via Brera 28, 20121 Milano, Italy \\ e-mail: marcella@brera.mi.astro.it \\ 2 INAF - Osservatorio Astronomico di Roma, via Frascati 33, 00040 Monte Porzio Catone, Italy \\ e-mail: [giallo, fontana]@coma.mporzio.astro.it \\ ${ }^{3}$ Laboratoire d'Astronomie de Marseille, Traverse du Siphon, BP 8, 13376 Marseille, France \\ e-mail: stephane.arnouts@oamp.fr \\ ${ }^{4}$ INAF - Osservatorio Astronomico di Trieste, via G. B. Tiepolo 11, 40131 Trieste, Italy \\ e-mail: [cristiani, nonino]@ts.astro.it \\ 5 European Southern Observatory, Karl-Schwarzschildstr. 2, 85748 Garching, Germany \\ e-mail: [sdodoric, evanzell]@eso.org \\ ${ }^{6}$ Dipartimento di Astronomia dell'Università di Padova, Vicolo dell'Osservatorio 2, 35122 Padova, Italy \\ Received 16 May 2003 / Accepted 25 February 2004

\begin{abstract}
A census of massive galaxies at redshift increasingly higher than $z \sim 1$ may provide strong constraints for the history of mass assembly and star formation. Here we report the analysis of three galaxies selected in the Hubble Deep Field South at $K \mathrm{~s} \leq 22$ on the basis of their unusually red near-IR color $J-K \geq 3$. We have used population synthesis models to constrain their redshifts and their stellar masses. One galaxy (HDFS-1269) is at redshift $z_{\text {phot }} \simeq 2.4$ while the other two (HDFS-822 and HDFS850) are at $z_{\text {phot }} \simeq 2.9-3.0$. All three galaxies have already assembled a stellar mass of about $10^{11} M_{\odot}$ at the observed redshift, placing the possible merging event of their formation at $z \gtrsim 3.5$. The inferred mass weighted age of their stellar populations implies that the bulk of the stars formed at $z_{\mathrm{f}}>3.5$. The resulting co-moving density of $\mathcal{M}_{\text {stars }} \gtrsim 10^{11} M_{\odot}$ galaxies at $\langle z\rangle \simeq 2.7$ is $\rho=1.2 \pm 0.7 \times 10^{-4} \mathrm{Mpc}^{-3}$, about a factor two higher than the predictions of hierarchical models. The comparison with the local density of galaxies implies that the three galaxies must have already formed most of their stellar mass and that they cannot follow an evolution significantly different from a passive aging. The comparison with the density of local $L \geq L^{*}$ early types (passively evolved galaxies) suggests that their co-moving density cannot decrease by more than a factor 2.5-3 from $z=0$ to $z \simeq 3$ suggesting that up to $40 \%$ of the stellar mass content of bright $\left(L \geq L^{*}\right)$ local early type galaxies was already in place at $z>2.5$.
\end{abstract}

Key words. galaxies: evolution - galaxies: elliptical and lenticular, cD - galaxies: formation

\section{Introduction}

Deep near-IR surveys are unveiling sources with unusually red near-IR colors $(J-K>3)$. They are extremely rare at magnitudes brighter than $K=20$ while their surface density increases at fainter magnitudes. Only one source redder than $J-K=3$ is present in the the $65 \operatorname{arcmin}^{2}$ surveyed by Hall et al. (2001) down to $K \simeq 19.5$ while 5 of them appear at $K \mathrm{~s} \leq 21$ over a sub-area of $43 \mathrm{arcmin}^{2}$ of the ESO Imaging Survey (Scodeggio \& Silva 2000). One red object (HDFN-JD1) was found in the Hubble Deep Field North (HDFN; Dickinson et al. 2000). It has a magnitude $K \simeq 22$ and no counterpart at wavelengths shorter than $1.2 \mu \mathrm{m}$. Maihara et al. (2001) and Totani et al. (2001) noticed the presence of four sources at magnitudes $K^{\prime} \gtrsim 21$ with color $J-K>3$ in the Subaru Deep Field

Send offprint requests to: P. Saracco, e-mail: saracco@brera.mi .astro.it
(SDF). Objects with these unusually red near-IR colors were also noticed in the HDF-S by Saracco et al. (2001) at $K>20.5$.

The nature of these sources has not yet been firmly established even if it is quite certain that they are not galactic objects. Indeed, very low mass stars, such as L-dwarfs, can display colors only slightly redder than $J-K=2$ (Chabrier et al. 2000; Kirkpatrick et al. 2000). Stars heavily reddened by circumstellar dust due to undergoing mass loss, such as Mira variables and carbon stars, can be redder than L-dwarfs. However, Whitelock et al. $(1995,2000)$ found only 2 stars having $J-K \simeq 3$ out of the 350 Mira and mass-losing stars observed. It seems unlikely that the extremely small fields of the HDFs and of the SDF can contain so many extremely rare stars at high galactic latitude. Furthermore, the apparent $K$-band magnitude of these unusually red objects $(K \gtrsim 20)$ would place them out of the Galaxy at a distance larger than $5 \mathrm{Mpc}$ if they were stars.

Cutri et al. (2001) and Smith et al. (2002) find 4 QSOs with colors $J-K>3$ among the 231 red AGNs selected using 
a $J-K>2$ criterion from the Two Micron All-Sky Survey (2MASS). They are brighter than $K=14$ and are at $z<0.3$. If the unusually red sources seen at $K \gtrsim 20$ were dominated by AGNs at these redshifts they would be at least $10^{3}$ times less luminous then the 2MASS AGNs, i.e. too faint to be AGNs. On the other hand, un-obscured AGNs at larger $z$ would become rapidly bluer since the rest-frame near-IR excess would be redshifted beyond the $K$-band. In the case of dust obscured AGNs, they should be reddened by at least $A_{V} \simeq 3 \mathrm{mag}$ and placed at $z \geq 2$ to match the observed $J-K$ color. However, such values of extinction characterize AGNs for which the rest-frame optical luminosities are usually dominated by the continuum of the host galaxy (e.g. Maiolino et al. 2000).

The Extremely Red Objects (EROs) studied so far (e.g. Thompson et al. 1999; Cimatti et al. 1999, 2002; Daddi et al. 2000; McCarthy et al. 2001; Martini et al. 2001; Mannucci et al. 2002; Miyazaki et al. 2002) are characterized by nearIR colors usually bluer than $J-K \simeq 2.5$. Colors redder than $J-K \sim 2.5$ are not expected even for passively evolved galaxies down to $z \sim 2$ (e.g. Saracco et al. 1999). Indeed, all the EROs spectroscopically observed so far lie at $z<2$ (e.g. Cimatti et al. 2002; Saracco et al. 2003). Thus, the unusually red near-IR color characterizing these objects suggests redshifts $z \gtrsim 2$ and, possibly, a component of dust absorption. Dickinson et al. (2000) consider various hypothesis for the nature of HDFN-JD1: from the most extreme of an objects at $z \gtrsim 10$, justified by the non-detection of the object from 0.3 to $1.1 \mu \mathrm{m}$, to the least extreme of a dusty galaxy at $z>2$ or a maximally old elliptical galaxy at $z \gtrsim 3$. The analysis of Hall et al. (2001) suggests a redshift $z \sim 2.4$ for their unusually red object. Totani et al. (2001), by comparing the $J-K$ color and the surface number density of the red sources in the SDF with model predictions, conclude that they are best explained by dusty elliptical galaxies at $z \sim 3$ in the starburst phase of their formation. Thus, the analysis of unusually red near-IR objects performed so far place these galaxies at $z>2-3$. Our knowledge of the Universe at these redshifts comes mostly from the Lyman-Break Galaxies (LBGs) selected through the U-dropout method based on UV-optical color (e.g. Steidel et al. 1996). The unusually red objects above are missed by this selection technique both due to their faintness at optical wavelength and to their different optical colors (see e.g. Vanzella et al. 2001). Consequently, also the information they bring relevant to the Universe at that $z$ are missed. For this reason they could be extremely important to probe the Universe at these redshifts.

In this paper we present the analysis based on a multi-band data set (from $0.3 \mu \mathrm{m}$ to $2.15 \mu \mathrm{m}$ ) of three $J-K \geq 3$ sources selected at $K \mathrm{~s} \leq 22$ on the HDF-S. The near-IR data have been collected by the Faint Infra-Red Extra-galactic Survey (FIRES, Franx et al. 2000). In Sect. 2 we present the imaging, the photometry and the spatial extent analysis of the three sources. In Sect. 3 we derive the redshift and, consequently, the stellar masses of the galaxies through the comparison of the data with population synthesis models. In Sect. 4 we derive the co-moving spatial density of these objects and we try to constrain their formation and evolution in Sect. 5. We summarize our results in Sect. 6. Throughout this paper, magnitudes are expressed in the Vega system unless explicitly statede otherwise. We adopt an $\Omega_{m}=0.3, \Omega_{\Lambda}=0.7$ cosmology with $H_{0}=70 \mathrm{~km} \mathrm{~s}^{-1} \mathrm{Mpc}^{-1}$.

\section{Imaging and photometric analysis}

The optical images are the version 2 of the Hubble Space Telescope images in the $F 300 W, F 450 W, F 606 W$ and $F 814 W$ filters $\left(U_{300}, B_{450}, V_{606}\right.$ and $I_{814}$ hereafter, Casertano et al. 2000). Near-IR images centered in the HDF-S come from the FIRES project (Franx et al. 2000) and they have been obtained with the ISAAC spectro-imager at VLT-Antu in the filters $J \mathrm{~s}, H$ and $K \mathrm{~s}$. The three sources analyzed here were originally noticed by Saracco et al. (2001) in the analysis of a first set of such data ( $\sim 8 \mathrm{~h}$ of exposure) and turned out to be EROs $(I-K>4)$ from the analysis of the multi-band data by Vanzella et al. (2001; object IDs: 822, 850 and 1269 hereafter). The photometry and the analysis presented here are based on the final FIRES data set (Labbé et al. 2003) summing up to about $\sim 30 \mathrm{~h}$ exposure per filter with a measured $F W H M \sim$ 0.55 arcsec. The near-IR data have been reduced following the recipe described in Saracco et al. (2001). Photometric redshifts and analysis of the whole multi-band data sample is presented in Fontana et al. (2003a). In Fig. 1, the optical $\left(B_{450}\right.$, $\left.V_{606}, I_{814}\right)$ and the near-IR $\left(J_{\mathrm{s}}, H\right.$ and $\left.K \mathrm{~s}\right)$ images of the three sources are shown. The intensity levels in each image are constrained within $-1 \sigma$ and $3 \sigma$ from the background. Colors have been measured within the $K$ s-band detection isophote by using Sextractor (Bertin \& Arnouts 1996) in double image mode. To this end, we first rebinned the IR images to the same pixel size and orientation of the WFPC2 images. Then, the WFPC2 images were smoothed to the same effective PSF of the IR images as described in Vanzella et al. (2001). Photometric errors have been obtained by rescaling the formal SExtractor errors by a correction factor to take into account the correlation of neighboring pixels. The procedure used to estimate the correction factor is described in Vanzella et al. (2001). None of the three sources has a detectable flux in the $U_{300}$-band, while only one of them (HDFS-1269) has a reliable flux in the $B_{450}$ band. The photometry we obtained is in good agreement with the one derived by Labbé et al. (2003). In Table 1 the optical and nearIR colors of the three sources are reported. It is worth noting that red AGNs have optical-IR colors 1.5-2 mag bluer than those of our sources (Francis et al. 2000; Smith et al. 2002). Thus, even if the presence of an AGN cannot be excluded (see e.g. van Dokkum et al. 2003), the optical-to-NIR colors exclude, once more, that the continuum emission is dominated by nuclear activity.

The three galaxies appear very compact in the $K$-band image. In Fig. 2 we compare the $K$-band surface brightness profile of the three galaxies with the profile of a bright star in the field. The two brightest galaxies (HDFS-1269 and HDFS-822) are clearly resolved while HDFS-850 is not resolved. Assuming that it is at a redshift comparable to the other two sources, as suggested by its colors, it is $\sim 1$ mag intrinsically fainter. Using the magnitude-size relations in Snigula et al. (2002) we found that, for the same profile, HDFS-850 would have a scale radius $\sim 1.6$ and $\sim 3$ times narrower than HDFS-822 (or HDFS-1269) in the case of an exponential and a de Vaucouleur 

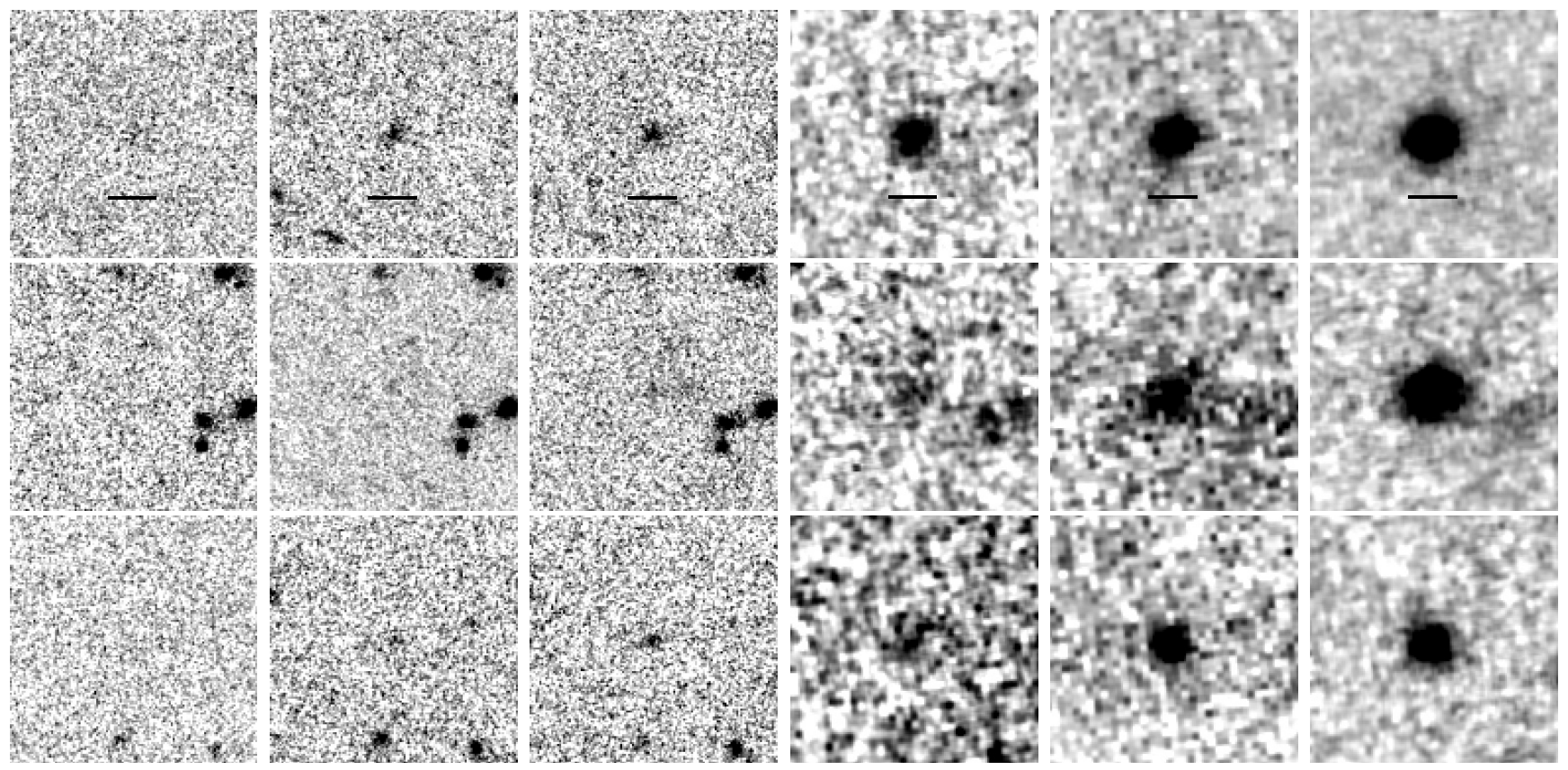

Fig. 1. From left to right: $B_{450}, V_{606}, I_{814}, J \mathrm{~s}, H$ and $K$ s band images of the three EROs 1269 (upper panel), 822 (middle panel) and 850 (lower panel). The images are $5 \times 5$ arcsec centered on the sources. The intensity of each image is in the range $[-1 \sigma ;+3 \sigma]$ from the background. The lines shown for reference in the images of 1269 are 1 arcsec width.

Table 1. Photometry of the three EROs 1269, 822 and 850. Magnitudes are in the Vega system. Colors are measured within the aperture defined by the $K$ s-band isophote. The $K$ s-band magnitude is the MAG_AUTO measured by Sextractor.

\begin{tabular}{lcccccccccc}
\hline \hline Object & $\mathrm{RA}(\mathrm{J} 2000)$ & $\operatorname{Dec}(\mathrm{J} 2000)$ & $U_{300}-K \mathrm{~s}$ & $B_{450}-K \mathrm{~s}$ & $V_{606}-K \mathrm{~s}$ & $I_{814}-K \mathrm{~s}$ & $J \mathrm{~s}-K \mathrm{~s}$ & $H-K \mathrm{~s}$ & $K \mathrm{~s}$ \\
\hline HDFS_1269 & $22: 32: 49.25$ & $-60: 32: 11.62$ & $>7.7$ & $7.6 \pm 0.5$ & $6.9 \pm 0.2$ & $5.5 \pm 0.1$ & $3.0 \pm 0.2$ & $1.2 \pm 0.1$ & $20.80 \pm 0.05$ \\
HDFS_822 & $22: 32: 53.39$ & $-60: 31: 54.33$ & $>7.6$ & $>7.6$ & $7.0 \pm 0.3$ & $5.7 \pm 0.2$ & $3.5 \pm 0.3$ & $1.4 \pm 0.2$ & $20.9 \pm 0.1$ \\
HDFS_850 & $22: 32: 53.01$ & $-60: 33: 57.03$ & $>6.8$ & $>6.8$ & $7.1 \pm 0.3$ & $5.6 \pm 0.2$ & $3.7 \pm 0.4$ & $1.1 \pm 0.2$ & $21.7 \pm 0.1$ \\
\hline
\end{tabular}

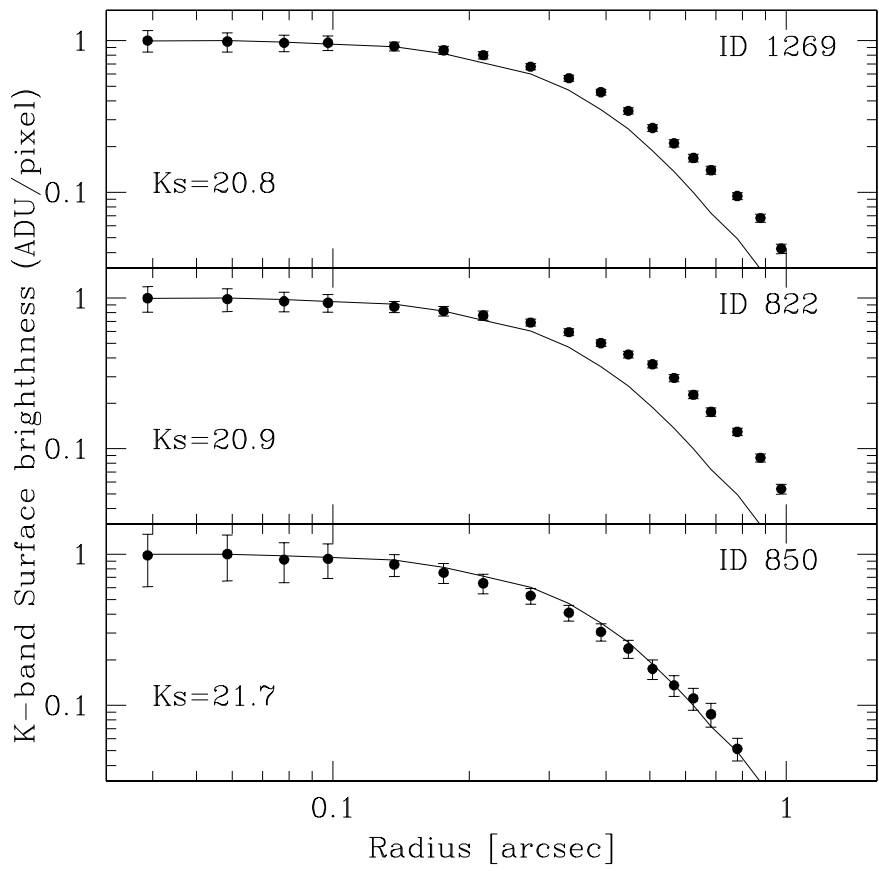

Fig. 2. Radial surface brightness profile of the three EROs discussed here (points) compared to one isolated star in the HDF-S (line). The profiles are normalized to the flux within the first aperture. profile respectively. Thus, considering the angular extent of the two brightest galaxies, it is expected that the profile of HDFS-850 is dominated by the seeing.

\section{Photometric redshifts and stellar masses of $J-K \geq 3$ galaxies}

In this section we compare the data with population synthesis models to constrain the redshift and the stellar mass of the three galaxies. The grid of templates used is based on the latest version of the Bruzual \& Charlot (1993) models and it is similar to the one described in Fontana et al. (2003a,b) in terms of star formation histories (SFHs), metallicity and extinction $\left(A_{V}\right)$. The SFHs considered, besides the simple stellar population (SSP) and the constant star formation rate (cst), are described by an exponentially declining star formation rate (SFR) with e-folding time $(\tau)$ in the range $0.1-15 \mathrm{Gyr}$. In this paper, besides the set of synthetic templates obtained with the Salpeter (Sal; Salpeter 1955) initial mass function (IMF; $0.1 M_{\odot}<\mathcal{M}<$ $\left.100 M_{\odot}\right)$, we considered also three analogous sets generated with the Miller-Scalo (MS; Miller \& Scalo 1979), Scalo (Sca; Scalo 1986) and Kroupa (Kro; Kroupa 2001) IMF respectively and a set based on the Kro IMF derived from the spectrophotometric models of Charlot \& Longhetti (2001). These latter take into account consistently the emission from stars and from 
Table 2. Parameters used to construct the grid of templates.

\begin{tabular}{ll}
\hline \hline SFH $\tau$ [Gyr] & $0.1,0.3,0.6,1,2,3,5,15$, SSP, $c s t$ \\
\hline Metallicity & $0.2 Z_{\odot}, 0.4 Z_{\odot}, Z_{\odot}$ \\
\hline$A \_V$ [mag] & $0 \div 2$ \\
\hline Extinction law & Calzetti et al. (2000) \\
\hline IMFs & Sal, Sca, MS, Kro, Kro+em \\
\hline
\end{tabular}

the gas (Kro+em) and allow to probe the possibility that the extreme near-IR colors are due to emission lines rather than to continuum emission (see e.g. Totani et al. 2001; Franx et al. 2003). The parameters used to generate the grid of synthetic templates are summarized in Table 2.

The $\chi^{2}$-minimization procedure of hyperz (Bolzonella et al. 2000) has been applied to find the best fitting spectral template to the observed colors for each set of IMF, SFH and metallicity. In practice, for a given IMF, metallicity and SFH, we find the best fitting template, i.e. the corresponding redshift and age. In the best fitting procedure the extinction has been allowed to vary within the range $0 \leq A_{V} \leq 2 \mathrm{mag}$ and, at each $z$, galaxies have been forced to have age lower than the Hubble time at that $z$. We repeated this procedure for the 5 IMFs, the 3 metallicity values and the 10 SFHs considered. Among the 150 best fitting templates thus obtained we considered those having a probability $\mathrm{P}\left(\tilde{\chi}^{2}\right)>0.68$ (where $\tilde{\chi}^{2}$ is the reduced $\chi^{2}$ ). For each galaxy, this set of templates has been used to define the range of variability of the relevant parameters we derived: $z$, SFHs and relevant age, stellar mass.

For each template considered, we derived the stellar mass $\mathcal{M}_{\text {stars }}$ of the galaxy from the mass-to- $K$ s-band light ratio since it is relatively insensitive to the star formation history with respect to the optical bands (Charlot 1996). The mass in this ratio (and thus $\mathcal{M}_{\text {stars }}$ ) results, in fact, by the integral of the SFR over the SFH of the template in the interval $0 \leq t \leq t_{\text {age }}$, where $t_{\text {age }}$ is the age of the template. This mass corresponds to the processed gas involved in the star forming process in this interval (see e.g. Madau et al. 1998). Thus, this is the mass that has been burned into stars at some time in this interval, i.e. the mass that was contained and/or is still locked into stars. We have also considered the mass locked into stars $\left(\mathcal{M}_{*}\right)$ at the age of the galaxy as resulting from the difference of $\mathcal{M}_{\text {stars }}$ and the processed gas returned to the interstellar medium through stellar winds. As far as our three galaxies, we have found that $\mathcal{M}_{*} \geq 0.8 \mathcal{M}_{\text {stars }}$ for Sal IMF and $\mathcal{M}_{*} \geq 0.6 \mathcal{M}_{\text {stars }}$ for MS IMF, this latter being the lowest fraction of mass locked into stars for the same value of $\mathcal{M}_{\text {stars }}$. The mass $\mathcal{M}_{*}$, is strongly related to the different mixture of stars and to their evolutionary stage. Thus, it is much more dependent on the IMF, the metallicity, the SFH and the extinction of the best fitting template with respect to $\mathcal{M}_{\text {stars }}$. For these reasons, we adopted $\mathcal{M}_{\text {stars }}$ as the stellar mass of the galaxies since it is a robust estimate.

The results of the best fitting procedure relevant to the Sal, MS and Kro IMFs are summarized in Table 3. We do not report the results obtained with the Sca IMF since the best fits obtained with this IMF were always worst than those obtained with the other IMFs and the values of the fitted parameters were within the ranges defined by the other models.
The results obtained with the set of templates including emission lines (Kro+em) are not listed in the table since the best fitting templates were always the same as those without emission lines (Kro). This latter result shows that the observed extremely red near-IR colors are not likely to be dominated by emission lines. In Table 4, we report for each galaxy the range of variability of the stellar mass $\left(\Delta \mathcal{M}_{\text {stars }}^{\mathrm{IMF}}\right)$ and of the mass-to- $K$ s-band light ratio $\left(\Delta M / L_{K}^{\mathrm{IMF}}\right)$ defined by the fitting models with different IMF.

In the following discussion of the individual objects, we compare the properties of local galaxies with those of our three galaxies. To this end, we derived a lower limit to their $K$ s-band luminosities at $z=0$ applying an evolutionary correction factor. The adopted correction factor for each galaxy is an upper limit to the expected luminosity evolution in the relevant range of redshift and corresponds to the passive aging of the youngest stellar population that could populate the galaxy at the relevant redshift. The age considered for the stellar population is the mass weighted age described in Sect. 5.1. The youngest age of each galaxy is chosen among the relevant best fitting templates $\left(P\left(\tilde{\chi}^{2}>0.68\right)\right)$. The passive aging is then traced from $z_{\text {phot }}$ to $z=0$ assuming a SSP with age at $z_{\text {phot }}$ equal to the youngest (mass weighted) age. It is worth noting that, for the relevant range of redshift, the expected luminosity evolution is not significantly dependent on the assumed IMF (differences less than $0.2 \mathrm{mag}$ ). We considered the $K$ s-band to minimize the uncertainties in this extrapolation. Indeed, the evolutionary corrections in the $K$ s-band are much smaller and less dependent on the SFH than those in the optical bands. For instance, the evolutionary correction for a passive aging from $z=3$ to $z=0$ in the case of a SSP is $\sim 1.7 \mathrm{mag}$ in $K \mathrm{~s}$ while it is $\sim 2.8 \mathrm{mag}$ in $V$.

HDFS_1269 - This is the sole object for which Sextractor has reliably detected flux in the $B_{450}$-band. The best-fitting value to the redshift of HDFS-1269 is $z_{\text {phot }}=2.4_{-0.2}^{+0.3}$. The quoted errors are the formal uncertainties of the fitting procedure ( $68 \%$ confidence level). The rest-frame (k-corrected) absolute magnitude of this galaxy, as resulting from its distant modulus (46.44) is $M_{K} \simeq-25.1$, i.e. $L>3 L^{*}$, where we considered $M_{K}^{*} \simeq-24.2$ for local galaxies (Cole et al. 2001; Huang et al. 2003). The best fit is formally given by a 2 Gyr old, $\tau=0.3$ Gyr model with an extinction $A_{V}=0.3$ and $Z=0.4 Z_{\odot}$ obtained with a Sal IMF (see Table 3). In Fig. 3 (upper panel) the bestfitting template is shown together with the photometric data points. For this galaxy 61 exponentially $\tau \geq 0.3$ Gyr decaying models fit the data $\left(0 \leq A_{V} \leq 2\right)$, providing Age in the range $1-4 \mathrm{Gyr}$ and redshifts $1.9<z<2.7$. The fitting models provide stellar masses of the order of $10^{11} M_{\odot}$ independently on the IMF assumed, as shown in Table 4. The stellar mass derived by the best fitting templates with the Sal IMF is $\mathcal{M}_{\text {stars }}=1.2 \times 10^{11} M_{\odot}$.

The degeneracy with respect to the SFH, does not help in tracing the evolution of this galaxy from $z_{\text {phot }}=2.4$ to $z=0$. Indeed, the stellar mass already assembled at $z=2.4$, may represent from $35 \%$ (if $\tau=15 \mathrm{Gyr}$ ) to almost $100 \%$ (if $\tau=0.3 \mathrm{Gyr}$ ) of the stellar mass the galaxy could form down to $z=0$, depending on the SFH considered among the 
Table 3. Parameters resulting from the best fits of stellar population models to the photometric data for Sal, MS and Kro IMFs. The $\tilde{\chi}^{2}$ represent the reduced $\chi^{2}$. The model which formally provides the best fit (the lowest $\tilde{\chi}^{2}$ ) is marked with “*”. The $V$-band absolute magnitude has been derived by the $K$ s-band apparent magnitude. The absolute magnitudes in the $K \mathrm{~s}$ and in the $V$ bands are corrected for the extinction.

\begin{tabular}{lllllllllllll}
\hline \hline ID & SFH & $\tilde{\chi}^{2}$ & $\mathrm{P}\left(\tilde{\chi}^{2}\right)$ & $z_{\text {phot }}$ & $\begin{array}{c}\text { Age } \\
{[\mathrm{Gyr}]}\end{array}$ & $\begin{array}{c}A_{V} \\
{[\mathrm{mag}]}\end{array}$ & $\begin{array}{c}Z \\
{\left[Z_{\odot}\right]}\end{array}$ & $\begin{array}{c}M_{V} \\
{[\mathrm{mag}]}\end{array}$ & $\begin{array}{c}M_{K} \\
{[\mathrm{mag}]}\end{array}$ & $\begin{array}{c}{ }^{a} M / L_{K} \\
{[M / L]_{\odot}}\end{array}$ & $\begin{array}{c}\mathcal{M}_{\text {stars }} \\
{\left[10^{11} M_{\odot}\right]}\end{array}$ \\
\hline Sal IMF & & & & & & & & & & \\
HDFS_1269* & $\tau=0.3$ & 0.33 & 0.92 & $2.4_{-0.2}^{+0.3}$ & 2.0 & 0.3 & 0.4 & -22.9 & -25.1 & 0.50 & 1.2 \\
HDFS_822 & $\tau=0.3$ & 0.06 & 0.99 & $3.0_{-0.2}^{+0.3}$ & 1.7 & 1.0 & 0.2 & -24.1 & -26.1 & 0.45 & 3.3 \\
HDFS_850 & $\tau=0.1$ & 0.16 & 0.98 & $2.9_{-0.3}^{+0.2}$ & 0.5 & 1.9 & 1.0 & -24.1 & -25.8 & 0.30 & 1.4 \\
\hline & MS IMF & & & & & & & & & & & 0.6 \\
HDFS_1269 & $\tau=0.3$ & 0.34 & 0.92 & $2.5_{-0.2}^{+0.3}$ & 2.0 & 0.2 & 0.4 & -22.9 & -25.1 & 0.26 & 0.2 \\
HDFS_822 & $\tau=0.3$ & 0.04 & 0.99 & $3.0_{-0.2}^{+0.3}$ & 1.7 & 0.9 & 0.2 & -24.1 & -26.1 & 0.23 & 1.4 \\
HDFS_850 & $\tau=0.1$ & 0.17 & 0.98 & $2.9_{-0.3}^{+0.2}$ & 0.5 & 1.8 & 1.0 & -24.0 & -25.8 & 0.14 & 0.6 \\
\hline & Kro IMF & & & & & & & & & & \\
HDFS_1269 & $\tau=0.3$ & 0.38 & 0.89 & $2.4_{-0.2}^{+0.3}$ & 2.0 & 0.4 & 0.4 & -22.9 & -25.1 & 0.38 & 0.9 \\
HDFS_822 & $\tau=0.3$ & 0.04 & 0.99 & $3.0_{-0.2}^{+0.3}$ & 1.7 & 1.0 & 0.2 & -24.1 & -26.0 & 0.35 & 2.1 \\
HDFS_850* & $\tau=0.1$ & 0.16 & 0.98 & $2.9_{-0.3}^{+0.2}$ & 0.5 & 1.9 & 1.0 & -24.0 & -25.8 & 0.22 & 1.0 \\
\hline
\end{tabular}

${ }^{a}$ We used $M_{\odot, K}=3.4$ (Allen 1973).

Table 4. Ranges of stellar masses $\left(\Delta \mathcal{M}_{\mathrm{stars}}^{\mathrm{IMF}}\right)$ and of mass-to- $K$ s-band light ratios $\left(\Delta M / L_{K}^{\mathrm{IMF}}\right)$ defined by the fitting models with different IMF (Sal, MS and Kro).

\begin{tabular}{lcccccc}
\hline \hline ID & $\Delta \mathcal{M}_{\text {stars }}^{\mathrm{Sal}}$ & $\Delta M / L_{K}^{\mathrm{Sal}}$ & $\Delta \mathcal{M}_{\text {stars }}^{\mathrm{MS}}$ & $\Delta M / L_{K}^{\mathrm{MS}}$ & $\Delta \mathcal{M}_{\text {stars }}^{\mathrm{Kro}}$ & $\Delta M / L_{K}^{\mathrm{Kro}}$ \\
& {$\left[10^{11} M_{\odot}\right]$} & {$[M / L]_{\odot}$} & {$\left[10^{11} M_{\odot}\right]$} & {$[M / L]_{\odot}$} & {$\left[10^{11} M_{\odot}\right]$} & {$[M / L]_{\odot}$} \\
\hline HDFS_1269 & $1.2-2.1$ & $0.35-0.75$ & $0.6-1.2$ & $0.20-0.44$ & $0.9-1.5$ & $0.29-0.49$ \\
HDFS_822 & $2.3-5.6$ & $0.25-0.52$ & $1.1-2.6$ & $0.14-0.27$ & $1.7-4.2$ & $0.2-0.47$ \\
HDFS_850 & $1.0-1.4$ & $0.25-0.45$ & $0.5-1.2$ & $0.12-0.24$ & $0.8-1.1$ & $0.18-0.28$ \\
\hline
\end{tabular}

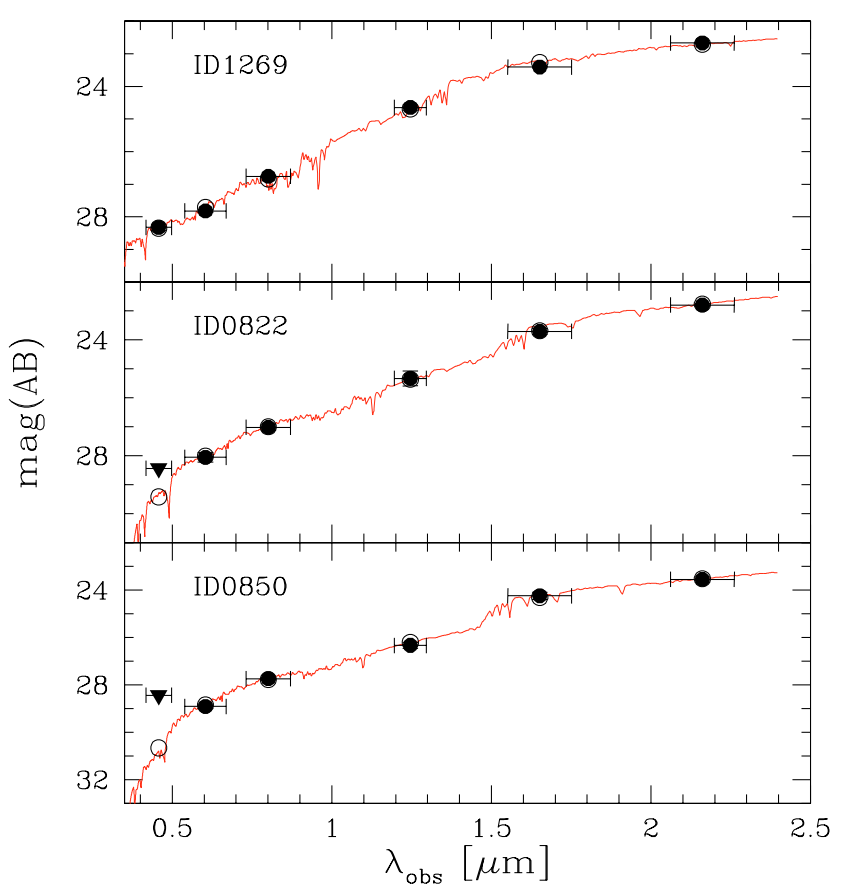

Fig. 3. The best fitting templates (see Table 3 ) are superimposed on the observed photometric data (filled points) in the $B_{450}, V_{606}, I_{814}, J \mathrm{~s}$, $H$ and $K$ s bands of the ERO 1269 (upper panel), 822 (middle panel) and 850 (lower panel) respectively. Magnitudes are in the AB system. Filled triangles represent upper limits. Open circles are the magnitudes derived from the best fitting template.
61 models. The lower limit to the $K$ s-band luminosity that this galaxy would have at $z=0$ is $L_{z=0} \geq L_{*}$, having estimated a passive evolutionary dimming of $\Delta M_{K} \simeq 1.3 \mathrm{mag}$ from $z_{\text {phot }}=2.4$ to $z=0$.

HDFS_822 - The best-fitting value to the redshift is $z_{\text {phot }}=$ $3.0_{-0.2}^{+0.3}$ and the resulting absolute magnitude is $M_{K} \simeq-26.0$ $\left(L>5 L^{*}\right)$. The best fit is formally given by a $1.7 \mathrm{Gyr}$ old, $\tau=0.3$ Gyr model with an extinction $A_{V}=0.9$ and $Z=0.2 Z_{\odot}$ obtained with a MS IMF and it is shown in Fig. 3 (middle panel). For this galaxy, 93 templates fit the data $\left(0.4 \leq A_{V} \leq 2\right)$ providing Age in the range 0.2-2.5 Gyr and redshifts $2.4<$ $z<3.2$ The stellar mass derived in the case of Sal IMF is $\mathcal{M}_{\text {stars }}=3.3 \times 10^{11} M_{\odot}$. For this galaxy the models provide a stellar mass always larger than $10^{11} M_{\odot}$ independently on the IMF assumed. Also in this case, the degeneracy with respect to the SFH implies that the stellar mass already formed at $z \simeq 3$ may represent from $20 \%$ to $\sim 100 \%$ of the stellar mass the galaxy could have at $z=0$. The lower limit to the $K$ s-band luminosity of this galaxy at $z=0$ is $L_{z=0}>1.2 L_{*}$ $\left(\Delta M_{K} \simeq 1.7 \mathrm{mag}\right)$.

HDFS_850 - This is the faintest and the reddest of the three galaxies selected. In this case the best-fitting template is given by a 0.5 Gyr old, $\tau=0.1$ Gyr model with an extinction $A_{V}=$ 1.9 and $Z=Z_{\odot}$ obtained with a Kro IMF. This model provides 
a redshift $z_{\text {phot }}=2.9_{-0.3}^{+0.2}$. For this ERO, 58 templates (all the $\tau \leq$ 2 Gyr models and SSP model) fit well the data with extinction $1 \leq A_{V} \leq 2$, Age in the range 0.25-1.7 Gyr and redshifts $2.6<$ $z<3.1$. The stellar mass derived is $\mathcal{M}_{\text {stars }}=1.4 \times 10^{11} M_{\odot}$ (Sal IMF). The stellar mass already formed and assembled at $z \simeq 2.9$ may represent from $\sim 70 \%$ to $\sim 100 \%$ of the stellar mass the galaxy could form down to $z=0$. The lower limit we derived to the $K$ s-band luminosity of this galaxy is $L_{z=0} \simeq L_{*}$ $\left(\Delta M_{K} \simeq 1.5 \mathrm{mag}\right)$.

Extremely young ages with active star formation and no dust attenuation are included among the best fitting templates. Hence, the minimum permissible mass-to-light ratio for each galaxy has been derived with the procedure adopted. Consequently, the lower bound of the range of variability of the stellar mass given in Table 4 should be considered a lower limit to the mass in stars of each galaxy. Moreover, active star formation can partially mask the presence of a more massive and much older stellar population (see e.g. Papovich et al. 2001) which could not be correctly accounted for using a single SFH as adopted in the present analysis. The results we obtained show that $i$ ) all three galaxies are at redshift $2<z<3$; ii) they have a high stellar mass content $\left(\mathcal{M}_{\text {stars }} \simeq 10^{11} M_{\odot}\right)$ already formed and assembled at the observed redshift; iii) they would populate the bright end ( $L_{z=0} \gtrsim L_{*}$ ) of the local luminosity function of galaxies even assuming they evolve passively.

\section{The density of $J-K \geq 3$ galaxies}

The co-moving spatial density of $J-K \geq 3$ galaxies and its statistical uncertainty have been estimated as

$\rho=\sum_{i} \frac{1}{V_{\max }^{i}}, \quad \sigma(\rho)=\left[\sum_{i}\left(\frac{1}{V_{\max }^{i}}\right)^{2}\right]^{1 / 2}$.

Thus, for each galaxy we computed the volume

$V_{\max }=\frac{\omega}{4 \pi} \int_{z_{1}}^{z_{\max }} \frac{\mathrm{d} V}{\mathrm{~d} z} \mathrm{~d} z$

where $\omega$ is the solid angle subtended by the HDF-S area and $z_{\max }$ is the maximum redshift at which each galaxy would be still included in the sample limited to $K s=22$. Since, for a given template the k-correction in $K \mathrm{~s}$ is nearly constant for $z>2-2.5$, in the derivation of the $z_{\max }$ of each galaxy we considered the average $\mathrm{k}$-correction derived by the best fitting templates at redshift $z=z_{\text {phot }}$. The lower bound in the integration $\left(z_{1}\right)$ should be defined by the $J-K>3$ color cut. In Fig. 4 the evolution of the $J-K$ color with redshift is shown for different SFHs and two different values of the extinction. It has been assumed that the star formation begins at $z_{\mathrm{f}}=6$ with a Sal IMF. The figure clearly shows that the $J-K>3$ color cut does not define a unique value of $z_{1}$ given the degeneracy with respect to the SFH and to the extinction. Thus, we assumed as $z_{1}$ the lower limit to the redshift of the lowest redshift galaxy (HDFS-1269), i.e. $z_{1}=1.9$. We find that at the average redshift $\langle z\rangle \simeq 2.7$ the co-moving density of galaxies brighter than $K \mathrm{~s}=22$ with $J-K \mathrm{~s} \geq 3$ is $\rho=1.2 \pm 0.7 \times 10^{-4} \mathrm{Mpc}^{-3}$.

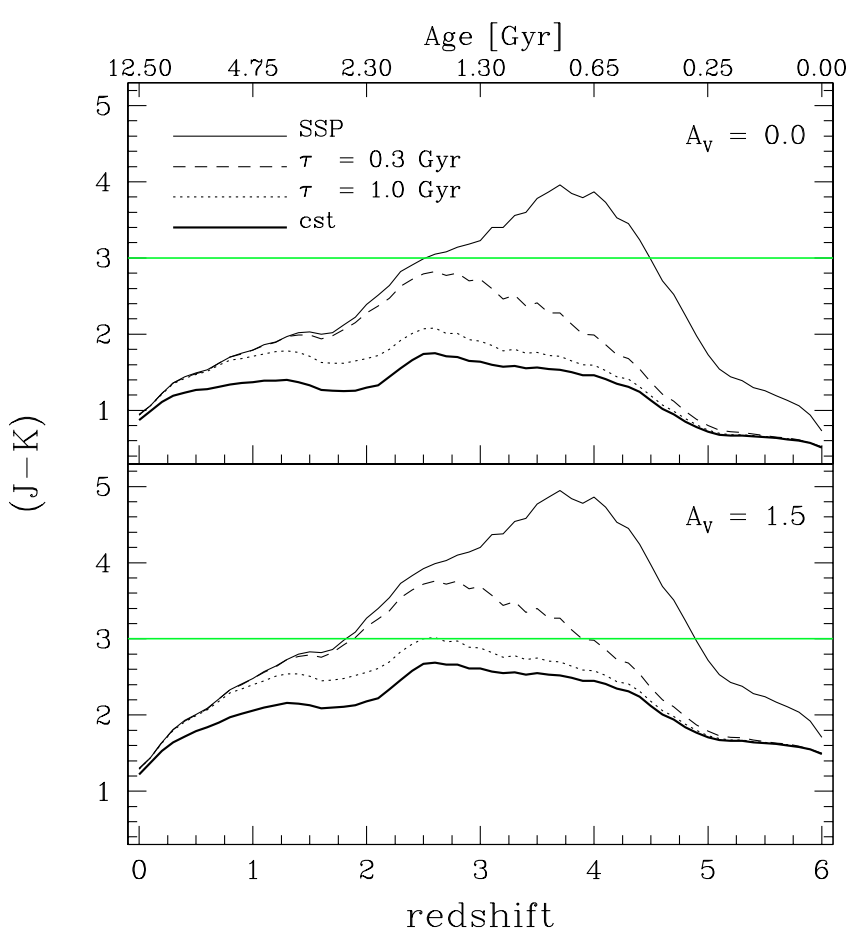

Fig. 4. Evolution of the $J-K$ color as a function of redshift. The different curves refer to the different SFHs quoted in the figure. All the models have been obtained with Sal IMF assuming a star formation beginning at $z_{\mathrm{f}}=6($ Age $=0)$ and two different values of extinction ( $A_{V}=0$ upper panel and $A_{V}=1.5$ lower panel). The age of the stellar population is shown on the top axis. The horizontal line defines the $J-K=3$ color cut used to select the three galaxies.

In the derivation of $z_{\max }$ we did not taken into account the surface brightness dimming which would tend to lower the maximum distance reachable. Moreover, galaxies with masses of about $10^{11} M_{\odot}$ at $z>2$ can escape the $J-K \mathrm{~s} \gtrsim 3$ threshold due to the uncertainties in the flux measure and to intrinsically bluer colors. Indeed, some of the galaxies selected by Labbé et al. (2003) with color $J-K>2.3$ at $K \mathrm{~s} \leq 22$ turned out to have redshift $z \gtrsim 2$ and masses of the order of $10^{11} M_{\odot}$ (Fontana et al. 2003b). Thus, it is likely that this density is an underestimate of the density of $\mathcal{M}_{\text {stars }} \gtrsim 10^{11} M_{\odot}$ galaxies at these redshifts.

\section{Constraining the evolution of massive galaxies}

In this section we attempt to constrain the possible evolutionary scenarios of the 3 massive galaxies. In Sect. 5.1 we concentrate on the formation and evolution of the stellar populations, while in Sect. 5.2 we consider the evolution of the number density of massive galaxies.

\subsection{Constraining the star formation history}

Spectrophotometric models, as those adopted in the present work, are not meant to describe the detailed evolutionary history of galaxies. Their aim is to describe the global observed photometric properties of a galaxy by means of an appropriate mix of stellar populations and different SFHs. Real galaxies are 
not necessarily expected to follow one of these SFHs. Different combinations of them can provide good fits to the photometric data of a given galaxy implying different behavior of its past and future evolution (as clearly shown in Sect. 3). Keeping these limits in mind, we have tried to constrain the SFH of the three massive galaxies.

In order to characterize the properties of their stellar populations we computed a more robust estimate of the age of the bulk of stars in the three EROs. Indeed, we cannot consider the age parameter provided by the best-fitting models given the degeneracy of the SFHs. We thus defined for each best fitting template a mass weighted age of the stars. Any template, defined by a fixed age and SFH, can be seen as the sum of SSPs with different ages. Each SSP provides the fraction of the total mass which depends on its own age and on the SFH describing the template itself. We derived the mass weighted age by summing the ages of the SSPs, each of them weighted on its mass fraction for each best-fitting template. The age of the bulk of stars in each galaxy is defined by the range spanned by the mass weighted age. Following this approach, we find that the bulk of the stars in HDFS-1269, HDFS-822 and HDFS-850 have ages $1.7 \pm 0.5 \mathrm{Gyr}, 1 \pm 0.6 \mathrm{Gyr}$ and $0.6 \pm 0.3 \mathrm{Gyr}$ respectively. Such estimates suggests that most of the stars have formed at $z_{\mathrm{f}} \gtrsim 3.5$ in all three galaxies. The obvious question is how the three galaxies have formed and/or assembled their stellar masses, i.e. how they evolved back in time.

Since in the hierarchical paradigm the age of the stars is not necessarily correlated with the epoch of assembly of the objects (Moustakas \& Somerville 2002), even our estimate of the age of the bulk of stars does not place any constraint on the past assembly history (if any) of the three galaxies. Thus, from the properties of the stellar populations of the three galaxies we cannot probe their possible formation through merging process. Anyway, their redshift $(z \sim 3)$ place the possible merging event at $z \gtrsim 3.5$ considering a dynamical time scale of $3 \times 10^{8} \mathrm{yr}$ (Mihos \& Hernquist 1996). On the other hand, we can probe the case in which the galaxies are the result of the SFHs resulting from the best-fitting procedure. This procedure produced a set of acceptable SFHs only on the basis of the photometry of the three galaxies. By means of the age of the stellar populations, the luminosity, the stellar mass and the comoving density of the three galaxies, we now try to bound further this set of SFHs. The following discussion and the results are, in fact, independent on the choice of the IMF. In order to obtain the ages of the bulk of stars estimated above in the three galaxies, the $\tau=0.1 \mathrm{Gyr}$ model (the lower bound of the SFHs) requires a formation redshift $z_{\mathrm{f}} \simeq 3.5$. To produce a stellar mass of about $10^{11} M_{\odot}$ within $0.1 \mathrm{Gyr}\left(3.3 \lesssim z_{\mathrm{f}} \lesssim 3.5\right)$, an average $S F R \simeq 1000 M_{\odot} \mathrm{yr}^{-1}$ is needed in this interval. Such high $S F R$ resembles that estimated by Ivison et al. (2002) for the 36 sources detected with SCUBA by Scott et al. (2002) and Fox et al. (2002) on $260 \mathrm{arcmin}^{2}$. Given the comoving density of the three galaxies $\left(\rho=1.2 \pm 0.7 \times 10^{-4} \mathrm{Mpc}^{-3}\right)$ and the volume sampled by the HDF-S within $3.3 \lesssim z_{\mathrm{f}} \lesssim 3.5$ $\left(\sim 3.1 \times 10^{3} \mathrm{Mpc}^{3}\right)$ we expect $0.4 \pm 0.2$ of these progenitors over an area equal to that of the HDF-S. This number would be consistent with the number of SCUBA sources expected on this area $(\sim 0.7)$ on the basis of the surface density measured by Scott et al. Star formation time scales longer than $1 \mathrm{Gyr}$ can in principle account as well for the stellar masses already formed and for the age of the bulk of stars, provided that $z_{\mathrm{f}}>5$ and $S F R \leq 100 M_{\odot} \mathrm{yr}^{-1}$. On the other hand, Fig. 4 suggests that a time scale longer would not be consistent with the observed $J-K$ color, unless to invoke an extinction in excess to $A_{V} \simeq 1.5-2 \mathrm{mag}$. However, even in this case, the star formation rate has to rapidly fade at $z \sim 3$ to match the constraints imposed by the local density of bright galaxies. Indeed, if the stellar mass already assembled $\left(\simeq 10^{11} M_{\odot}\right)$ by the three galaxies at $z_{\text {phot }}$ was lower than $50 \%$ of the total mass in stars they could form from $z_{\mathrm{f}}$ to $z=0$, they would be brighter than $2-3 L^{*}$ at $z=0$. Thus, at $z=0$ we should measure a density of galaxies brighter than 2-3 $L^{*}$ not lower than $1.2 \times 10^{-4}$. The local density of galaxies brighter than $2 L^{*}$ and $3 L^{*}$ is $1.8 \times 10^{-4} \mathrm{Mpc}^{-3}$ and $0.5 \times 10^{-4} \mathrm{Mpc}^{-3}$ respectively, i.e. comparable or lower than the density of the three galaxies. Thus, the photometry, the stellar masses already formed and assembled, the mean ages of the stellar populations together with the constraints imposed by the local density of bright galaxies, suggest that the three galaxies are consistent with galaxies which at $z \sim 3$ have formed most of their stellar mass and which will follow a passive aging in time soon after few Myrs, i.e. with a massive early type galaxy.

\subsection{Constraining the density evolution}

We have compared the density of local massive galaxies with that at redshift $z \simeq 2.7$. We have previously found that the luminosity of the three galaxies at $z=0$ has to be $\sim L^{*}$ in the case of passive evolution and should not be brighter than $\sim 2 L^{*}$ in the case of a slightly longer star formation activity. In this latter hypothesis, the three galaxies would represent $\sim 60 \%$ of the whole population of massive local galaxies $\left(L \geq 2 L^{*}\right)$. In the hypothesis of passive aging, we have to consider the local number density of early type galaxies. By integrating the LF of E/S0 galaxies brighter than $L^{*}\left(\mathcal{M}_{\text {star }} \simeq 10^{11} M_{\odot}\right)$ we obtained a density of $3 \times 10^{-4} \mathrm{Mpc}^{-3}$, where we used $\phi_{\mathrm{E} / \mathrm{S} 0}^{*}=1.5 \times 10^{-3} \mathrm{Mpc}^{-3}$ (Marzke et al. 1998). Thus, the three galaxies account for about $40 \%$ of the local population of early type galaxies. This result implies also that $\sim 40 \%$ of the stellar mass content of local early type galaxies was already in place at $z \gtrsim 2.5$ and that their number density cannot decrease by more than a factor $2.5-3$ from $z=0$ to $z \simeq 3$. In the semi-analytical rendition of hierarchical models by Kauffmann \& Charlot (1998a,b), the predicted number density of $\mathcal{M}_{\text {star }} \geq$ $10^{11} M_{\odot}$ ellipticals decreases by a factor $\sim 8$. Indeed, the predicted density of $\mathcal{M}_{\text {star }} \geq 10^{11} M_{\odot}$ ellipticals at $z \simeq 2.5$ is about 5-6 $\times 10^{-5} \mathrm{Mpc}^{-3}$, a factor 2 lower than our estimate. The same conclusion is reached considering the predictions of Moustakas \& Somerville (2002). They predict a number density of halos hosting local bright $\left(L>L^{*}\right)$ elliptical galaxies (gEs) at $z \simeq 2.5$ more than a factor two lower than our estimate. The semi-analytic models of galaxy formation in the hierarchical clustering scenario by Baugh et al. $(1998,2002)$ predict, in fact, no such massive galaxies at redshift $z>2$. These results are summarized in Fig. 5. The figure suggests that the evolution 


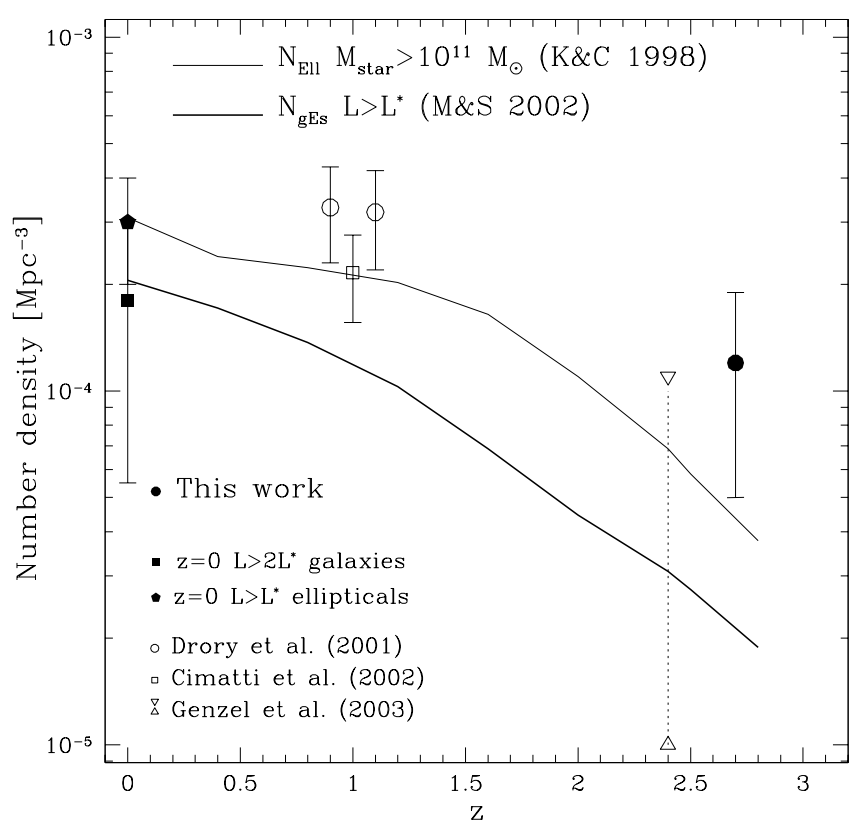

Fig. 5. Number density of galaxies as a function of redshift. The filled circle is our estimate of the density of $\mathcal{M}_{\text {stars }} \simeq 10^{11} M_{\odot}$ galaxies at $z \simeq 2.7$. The filled triangle is the local number density of $L>2 L^{*}$ galaxies obtained by integrating the LF of Cole et al. (2001; see Sect. 4). The filled pentagon is the density of local $L>L^{*}$ early type galaxies derived by the LF of Marzke et al. (1998). The open square is the density of $L \gtrsim 0.3 L^{*}$ ellipticals from Cimatti et al. (2002) and the open circles are the upper limit to the density of $\mathcal{M}_{\text {stars }} \geq 10^{11} M_{\odot}$ galaxies from Drory et al. (2001) (see text for details). The two open triangles connected by the dotted line represent the range of values of the density of $\mathcal{M}_{\text {stars }}>10^{11} M_{\odot}$ galaxies as derived by Genzel et al. (2003) from the SCUBA lens survey. The thin line is the density evolution of ellipticals with stellar mass greater than $\mathcal{M}_{\text {stars }}=10^{11} M_{\odot}$ from Kauffmann \& Charlot (1998a,b; K\&C 1998); the thick line is the density evolution of dark matter halos hosting present-day bright $\left(L>L^{*}\right)$ ellipticals (gEs) from Moustakas \& Somerville (2002; M\&S 2002).

of the number density of massive $\left(\mathcal{M}_{\text {stars }} \simeq 10^{11} M_{\odot}\right)$ galaxies with redshift is slower than that predicted by the current hierarchical models, at least in the redshift range $0<z<3$. It is worth noting that, by assuming a value of $\mathcal{M}_{\text {star }} \simeq 10^{11} M_{\odot}$ for the three EROs, we derive at $z \simeq 2.7$ a stellar mass density $\rho_{\text {star }}=1.2( \pm 0.7) \times 10^{7} \mathrm{M}_{\odot} \mathrm{Mpc}^{-3}$. This density is $\sim 30 \%$ of the total stellar mass at this redshift (Fontana et al. 2003b).

The density we estimated is lower than the density of ellipticals $\left(2.16 \pm 0.6 \times 10^{-4} \mathrm{Mpc}^{-3}\right)$ spectroscopically identified at $0.85<z<1.3$ by Cimatti et al. (2002). On the other hand, this estimate includes mainly ellipticals less massive than $10^{11} M_{\odot}$. Indeed, given the limiting magnitude $K=19.2$ of their spectroscopic sample, they are sampling the counterpart of local $L \gtrsim 0.3 L^{*}\left(\mathcal{M}_{\text {star }}>10^{10} M_{\odot}\right)$ ellipticals in that redshift range. Our estimate is also lower than the density of $\mathcal{M}_{\text {star }} \geq 10^{11} M_{\odot}$ galaxies at $z \sim 1$ estimated by Drory et al. (2001). This is expected since they use an approach which maximizes the stellar mass of the galaxies for any $K$-band luminosity at any redshift. Thus their estimate represent the upper limit of $\mathcal{M}_{\text {star }} \geq 10^{11} M_{\odot}$ galaxies at that $z$.

\section{Discussion and conclusions}

We have presented the analysis of the three galaxies selected on the HDF-S on the basis of their unusually red $(J-K \geq 3)$ color. We have constrained their redshifts and estimated their stellar mass content by comparing the photometric data with population synthesis models obtained for different IMFs, SFHs, metallicity and extinction values. The grid of templates we used includes a set of template derived from the spectral models of Charlot \& Longhetti (2001) which take into account consistently the emission from stars and from the gas. This has allowed us to exclude the possibility that the observed extreme colors are dominated by emission lines. We find that one of the galaxies (HDFS-1269) is at $z_{\text {phot }} \simeq 2.4\left(P\left(\chi^{2}\right)=0.92\right)$ while the other two (HDFS-822 and HDFS-850) are at $z_{\text {phot }} \simeq 3$ $\left(P\left(\chi^{2}\right) \geq 0.98\right)$. All three galaxies have already assembled a stellar mass of about $10^{11} M_{\odot}$ at the relevant redshift. The fact that the two galaxies at $z \sim 3$ have assembled this mass places the possible merging event of their formation at $z \gtrsim 3.5$ considering a dynamical time scale of $3 \times 10^{8} \mathrm{yr}$ (e.g. Mihos \& Hernquist 1996). The inferred mass weighted age of the stellar populations places the formation of their bulk at $z_{\mathrm{f}}>3.5$ in all three galaxies suggesting a substantial amount of star formation at these redshifts in addition to the one derived by LBGs (e.g. Ferguson et al. 2002). Galaxies with stellar masses $\mathcal{M}_{\text {star }} \gtrsim 10^{11} M_{\odot}$ fully assembled at $z>2$ were previously found by other authors. For instance, Francis et al. (2001) find two luminous extremely red galaxies at $z \sim 2.4$ whose radial profiles suggest they are elliptical galaxies. They estimate a mass $\sim 10^{11} M_{\odot}$ and an age of $\sim 7 \times 10^{8} \mathrm{yr}$ for their stellar populations. Shapley et al. (2001) find some galaxies with such high stellar masses and old ages among their LBGs at $z \simeq 3$. Genzel et al. (2003) estimate a conservative lower limit of $1.4 \times 10^{11} M_{\odot}$ to the stellar mass of the sub-mm source at $z \simeq 2.8$ they studied. Contrary to these and our findings, Dickinson et al. (2003) do not find galaxies more massive than $10^{11} M_{\odot}$ at $z>2$ in the HDFN. The small area covered by the HDFs could be the reason of this discrepancy even if surface densities of $J-K \gtrsim 3$ galaxies comparable or higher than that in the HDF-S are found in the other pencil beams reaching similar depth (e.g. Bershady et al. 1998; Totani et al. 2001).

We found that the three galaxies must have already formed most of their stellar mass and that they cannot follow an evolution significantly different from a passive aging. This suggests that $J-K>3$ galaxies are most likely in the post starburst phase rather than in the starburst phase of their formation as hypothesized by Totani et al. (2001). These findings strongly support the thesis that $J-K>3$ galaxies are the high $-z$ counterpart of local $\mathcal{M}_{\text {star }} \gtrsim 10^{11} M_{\odot}$ early type galaxies and agree with the recent finding of an increasing clustering of high- $z$ galaxies with redder colors (Daddi et al. 2003; Roche et al. 2003). We estimated a co-moving density of galaxies brighter than $K \mathrm{~s}=22$ and redder than $J-K=3$ $\rho=(1.2 \pm 0.7) \times 10^{-4} \mathrm{Mpc}^{-3}$ at the average redshift $\langle z\rangle \simeq 2.7$ which should be considered an underestimate of the number density of galaxies with $\mathcal{M}_{\text {star }} \gtrsim 10^{11} M_{\odot}$ at that $z$. This value is about a factor two higher than the predictions of hierarchical models renditions by Kauffmann \& Charlot (1998a,b) 
and Moustakas \& Somerville (2002). In the hypothesis of passive evolution, their luminosities at $z=0$ would be $L \geq L^{*}$. By comparing the density of local $L \geq L^{*}$ early type galaxies with our estimate we find that their density cannot decrease by more than a factor $2.5-3$ from $z=0$ to $z \simeq 3$ suggesting that up to $40 \%$ of the stellar mass contained in local massive galaxies was already in place at $z \gtrsim 2.5$.

Acknowledgements. This work is based on observations made with the ESO-VLT telescopes at Paranal Observatory under program ID 164.O-0612 and with the NASA/ESA Hubble Space Telescope, obtained from the data archive at the Space Telescope Institute. STScI is operated by the association of Universities for Research in Astronomy, Inc. under the NASA contract NAS 5-26555.

\section{References}

Allen, C. W. 1973, Astrophysical Quantities (London: Athlon Press)

Baugh, C. M., Benson, A. J., Cole, S., Frenk, C. S., \& Lacey, C. 2002 [arXiv: astro-ph/0203051]

Baugh, C. M., Cole, S., Frenk, C. S., \& Lacey, C. 1998, ApJ, 498, 504 Bershadyh, M. A., Lowenthalh, J. D., \& Koo, D. C. 1998, ApJ, 505, 50

Bertin, E., \& Arnouts, S. 1996, A\&AS, 117, 393

Bolzonella, M., Miralles, J.-M., \& Pellò, R. 2000, A\&A, 363, 476

Bruzual, A. G., \& Charlot, S. 1993, ApJ, 405, 538

Calzetti, D., Armus, L., Bohlin, R. C., et al. 2000, ApJ, 533, 68

Casertano, S, de Mello, D., Dickinson, M., et al. 2000, AJ, 120, 2747

Charlot, S. 1996, in The Universe at High- $z$, Large Scale Structure and the Cosmic Microwave Background, ed. E. Martinez-Gonzalez, \& J. L. Sanz (Heidelberg-Springer), 53

Charlot, S., \& Longhetti, M. 2001, MNRAS, 323, 887

Chabrier, G., Baraffe, I., Allard, F., \& Haushildt, P. 2000, ApJ, 542, 464

Cimatti, A., Daddi, E., di Serego Alighieri, S., et al. 1999, A\&A, 352, L45

Cimatti, A., Daddi, E., Mignoli, M., et al. 2002, A\&A, 381, L68

Cole, S., Norberg, P., Baugh, C., et al. 2001, MNRAS, 326, 255

Cutri, R. M., Nelson, B. O., Kirkpatrick, J. D., Huchra, J. P., \& Smith, P. S. 2001, ASP Conf. Ser., 232, 78

Daddi, E., Cimatti, A., Pozzetti, L., et al. 2000, A\&A, 361, 535

Daddi, E., Rottgering, H. J. A., Labbé, I., et al. 2003, ApJ, 588, 50

Dickinson, M., Hanley, C., Elston, R., et al. 2000, ApJ, 531, 624

Dickinson, M., Papovich, C., Ferguson, H. C., \& Budavari, T. 2003, ApJ, 587, 25

Drory, N., Bender, R., Snigula, J., et al. 2001, ApJ, 562, L111

Ferguson, H. C., Dickinson, M., \& Papovich, C. 2002, ApJ, 569, L68

Fontana, A., et al. 2003a, in preparation

Fontana, A., Donnarumma, I., Vanzella, E., et al. 2003b, ApJL, submitted

Fox, M. J., Efstathiou, A., Rowan-Robinson, M., et al. 2002, MNRAS, 331,839

Francis, P. J., Whiting, M. T., \& Webster, R. L. 2000, PASA, 53, 56

Francis, P. J., Williger, G. M., Collins, N. R., et al. 2001, ApJ, 554, 1001
Franx, M., Moorwood, A., Rix, H.-W., et al. 2000, Messenger, 99, 20 Franx, M., Labbé, I., Rudnick, G., et al. 2003, ApJ, 587, L79

Genzel, R., Baker, A. J., Tacconi, L. J., et al. 2003, ApJ, 584, 633

Hall, P. B., Sawicki, M., Martini, P., et al. 2001, AJ, 121, 1840

Huang, J.-S., Glazebrook, K., Cowie, L. L., \& Tinney, C. 2003, ApJ, 584, 203

Ivison, R. J., Greve, T. R., Smail, I., et al. 2002, MNRAS, 337, 1

Kauffmann, G., \& Charlot, S. 1998a, MNRAS, 297, L23

Kauffmann, G., \& Charlot, S. 1998b [arXiv: astro-ph/9810031]

Kirkpatrick, J. D., Reid, I. N., Liebert, J., et al. 2000, AJ, 120, 447

Kroupa, P. 2001, MNRAS, 322, 231

Labbé, I., Franx, M., Rudnick, G., et al. 2003, AJ, 125, 1107

Madau, P., Pozzetti, L., \& Dickinson, M. 1998, ApJ, 498, 106

Maihara, T., Iwamuro, F., Tanabe, H., et al. 2001, PASJ, 53, 25

Maiolino, R., Salvati, M., Antonelli, L. A., et al. 2000, A\&A, 355, L47

Mannucci, F., Pozzetti, L., Thompson, D., et al. 2002, MNRAS, 329, L57

Martini, P. 2001, AJ, 121, 598

Marzke, R. O., Da Costa, L. N., Pellegrini, P. S., Willmer, C. N. A., \& Geller, M. J. 1998, ApJ, 503, 617

McCarthy, P. J., Carlberg, R. G., Chen, H.-W, et al. 2001, ApJ, 560, L131

Mihos, J. C., \& Hernquist, L. 1996, ApJ, 464, 641

Miller, G. E., \& Scalo, J. M. 1979, ApJS, 41, 513

Miyazaki, M., Shimasaku, K., Kodama, T., et al. 2002, PASJ, 55, 1079

Moustakas, L. A., \& Somerville, R. S. 2002, ApJ, 577, 1

Papovich, C., Dickinson, M., \& Ferguson, H. C. 2001, ApJ, 559, 620

Poli, F., Giallongo, E., A., Fontana, et al. 2003, ApJL, submitted

Roche, N. D., Dunlop, J., \& Almaini, O. 2003, MNRAS, 346, 803

Rudnick, G., Franx, M., Rix, H.-W., et al. 2001, AJ, 122, 2205

Salpeter, E. E. 1955, ApJ, 121161

Saracco, P., D’Odorico, S., Moorwood, A. F. M., et al. 1999, A\&A, 349,751

Saracco, P., Giallongo, E., Cristiani, S., et al. 2001, A\&A, 375, 1

Saracco, P., Longhetti, M., Severgnini, P., et al. 2003, A\&A, 398, 127

Scalo, J. M. 1986, Fundamentals of Cosmic Physics, 11, 1

Scodeggio, M., \& Silva, D. R. 2000,A\&A, 359, 953

Scott, S. E., Fox, M. J., Dunlop, J. S., et al. 2002, MNRAS, 331, 817

Shapley, A. E., Steidel, C. C., Adelberger, K. L., et al. 2001, ApJ, 562, 95

Smith, P. S., Schmidt, G. D., Hines, D. C., Cutri, R. M., \& Nelson, B. O. 2002, ApJ, 569, 23

Snigula, J., Drory, N., Bender, R., et al. 2002, MNRAS, 336, 1329

Steidel, C. C., Giavalisco, M., Pettini, M., Dickinson, M., \& Adelberger, K. L. 1996, ApJ, 462, L17

Thompson, D., Beckwith, S. V. W., Fockenbrock, R., et al. 1999, ApJ, 523, 100

Totani, T., Yoshii, Y., Iwamuro, F., Mahiara, T., \& Motohara, K. 2001, ApJ, 558, L87

Vanzella, E., Cristiani, S., Saracco, P., et al. 2001, AJ, 122, 2190

van Dokkum, P. G., Forster Schreiber, N. M., Franx, M., et al. 2003, ApJ, 587, L83

Whitelock, P., Menzies, J., Feast, M., et al. 1995, MNRAS, 276, 219

Whitelock, P., Marang, F., \& Feast, M. 2000, MNRAS, 319, 728 